\title{
Oxidized S100A4 inhibits the activation of protein phosphatase 5 through S100A1 in MKN-45 gastric carcinoma cells
}

\author{
MITSUMASA TSUCHIYA ${ }^{1 *}$, FUMINORI YAMAGUCHI ${ }^{2 *}$, SEIKO SHIMAMOTO $^{1}$, TOMOHITO FUJIMOTO ${ }^{1}$, \\ HIROSHI TOKUMITSU ${ }^{1}$, MASAAKI TOKUDA ${ }^{2}$ and RYOJI KOBAYASHI ${ }^{1}$ \\ Departments of ${ }^{1}$ Signal Transduction Sciences and ${ }^{2}$ Cell Physiology, \\ Kagawa University Faculty of Medicine, Kagawa 761-0793, Japan
}

Received March 8, 2014; Accepted September 23, 2014

DOI: $10.3892 /$ ijmm.2014.1947

\begin{abstract}
S100 proteins bind to numerous target proteins, as well as other S100 proteins and activate signaling cascades. S100 proteins can be modified by various post-translational modifications, such as phosphorylation, methylation and acetylation. In addition, oxidation is important for modulating their activities. Previous studies have shown that S100A1 interacts with S100A4 in vitro and in vivo. Due to this potential cross-talk among the S100 proteins, the aim of the present study was to examine whether S100A4 modulates the activity of S100A1. S100A4 was readily oxidized and formed disulfide-linked dimers and oligomers. Although non-oxidized S100A4 bound to protein phosphatase 5 (PP5), the $\mathrm{Cu}$-oxidized S100A4 failed to bind PP5. Instead, the $\mathrm{Cu}$-oxidized S100A4 directly interacted with S100A1 and prevented PP5 activation. Hydrogen peroxide induced S100A4 oxidation in MKN-45 gastric adenocarcinoma cells and decreased S100A1-PP5 interaction, resulted in the inhibition of PP5 activation by S100A1. These data indicate that oxidized S100A4 regulates PP5 activity in a unique manner under oxidative stress conditions.
\end{abstract}

Correspondence to: Professor Ryoji Kobayashi, Department of Signal Transduction Sciences, Kagawa University Faculty of Medicine, 1750-1 Ikenobe, Kagawa 761-0793, Japan

E-mail: ryoji@med.kagawa-u.ac.jp

*Contributed equally

Abbreviations: ASK1, apoptosis stimulating kinase 1; CaM, calmodulin; CBB, Coomassie brilliant blue; DTT, dithiothreitol; $\mathrm{H}_{2} \mathrm{O}_{2}$, hydrogen peroxide; HMW, higher molecular weight; His, 6xHistidine; HRP, horseradish peroxidase; NF- $\kappa \mathrm{B}$, nuclear factor- $\kappa \mathrm{B}$; PBS, phosphate-buffered saline; PP5, protein phosphatase 5; PPP, phosphoprotein phosphatase; ROS, reactive oxygen species; SPR, surface plasmon resonance; TNF- $\alpha$, tumor necrosis factor $\alpha$; TPR, tetratricopeptide repeat; Tricine, N-[2-hydroxy-1,1-bis (hydroxymethyl) ethyl] glycine; TTBS, Tris-buffered saline with $0.05 \%$ Tween- 20

Key words: oxidative stress, S100 protein, protein phosphatase 5, MKN-45

\section{Introduction}

S100A4 protein is a member of the EF-hand calcium ion-binding protein family, of which $>25$ members have been found in humans (1). They are 25-65\% homologous at the amino acid level, while the sequence of the linker (hinge) region and the $\mathrm{C}$-terminal extension are the most variable among the S100 proteins. Each S100 monomer contains two EF-hand $\mathrm{Ca}^{2+}$-binding sites. $\mathrm{Ca}^{2+}$-binding causes a conformational change and exposure of a hydrophobic surface allowing interaction with target proteins (2). They do not possess enzymatic activity, but rather regulate the activity of target proteins. Several proteins have been identified as S100A4 targets, including liprin $\beta 1$ (3), methionine aminopeptidase (4), the p53 tumor suppressor protein (5) and the heavy chain of non-muscle myosin II (6). In addition, S100A4 is capable of forming heterodimers with S100A1 $(7,8)$, which is likely to increase its functional potential. Previous studies have shown that S100A4 interacts with S100A1 via heterodimer formation and that S100A1 can antagonize function of S100A4 $(9,10)$. It has been a general observation that two-hybrid screenings utilizing S100 proteins have primarily detected other S100 family members as targets (11-13).

We have previously demonstrated that S100A1, A2, A6 and S100B interact with the tetratricopeptide repeat (TPR) domain of PP5 in a $\mathrm{Ca}^{2+}$-dependent manner and significantly activate its phosphatase activity (14). PP5 is a member of the phosphoprotein phosphatase (PPP) family of a serine/threonine phosphatase (15). PP5 contains a C-terminal catalytic domain and $\mathrm{N}$-terminal three TPR motifs that are unique in the PPP family $(16,17)$. The TPR motif consists of 34 amino acid sequences, and between 3 and 16 copies of the motif are arranged in the proteins as tandem arrays $(18,19)$. This motif can act as an interaction scaffold for protein complex formation. PP5 is a negative regulator of the apoptosis stimulating kinase 1 (ASK1) and modulates the apoptosis under oxidative stress conditions (20). Gastric epithelium is constantly exposed to reactive oxygen species (ROS) and activation of ASK1 by Helicobacter pylori under oxidative stress was reported previously (21).

The purpose of the present study was to investigate whether S100A4 affects S100A1 function (i.e., PP5 activation) under oxidative conditions. The oxidized form of S100A4, but not 
the native S100A4 dimer, was found to inhibit the activation of PP5 by S100A1 in vitro and in MKN-45 cells.

\section{Materials and methods}

Materials. Nickel-nitrilotriacetic acid-agarose was purchased from Qiagen (Hilden, Germany). Rabbit anti-S100A1 antibody (NB100-91955) was obtained from Novus Biologicals (Littleton, CO, USA). Sheep anti-S100A4 antibody (AF4138) was obtained from R\&D Systems (Minneapolis, MN, USA). Mouse anti-FLAG-horseradish peroxidase (HRP) antibody (A8592) and other chemicals were purchased from Sigma (St. Louis, MO, USA). Goat-anti-rabbit IgG- HRP-linked antibody (7074) was obtained from Cell Signaling (Beverly, MA, USA) and Donkey-anti-sheep IgG-HRP-conjugated antibody (HAF016) was purchased from R\&D Systems.

Plasmids and recombinant proteins. Human PP5 [GenBank: NM_006247] was cloned to pET16a and pME18S-FLAG vector, and rat S100A1 [Genbank: NM_001007636] and rat S100A4 [Genbank: NM_012618] were cloned to pET11a plasmids as previously reported (14). Histidine-tagged PP5 (His-PP5) protein was expressed and purified according to the manufacturer's instructions. S100A1 and S100A4 proteins were expressed and purified as previously described $(22,23)$. Purified recombinant S100A4 proteins were diluted $(0.5 \mathrm{mg} / \mathrm{ml})$ and maintained at $-30^{\circ} \mathrm{C}$ in a freezer for 3 months (air-oxidized). $\mathrm{Cu}$-oxidized S100A4 protein was prepared in accordance with a previous study (24). Briefly, S100A4 $(10 \mu \mathrm{M})$ was incubated

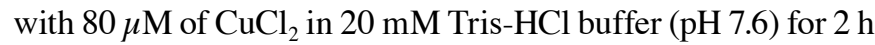
at $37^{\circ} \mathrm{C}$. Subsequent to stopping the reaction with the copper chelator diethylenetriamine-N,N,N',N',N'-pentaacetic acid, the product solution was desalted using centriprep-3 (Amicon, Inc., Beverly, MA, USA), dialyzed against $20 \mathrm{mM}$ Tris-HCl buffer ( $\mathrm{pH}$ 7.6). For Tricine SDS-PAGE analysis, samples were treated with (reducing conditions) or without (non-reducing conditions) $10 \mathrm{mM}$ dithiothreitol (DTT) prior to electrophoresis.

Cell culture and $\mathrm{H}_{2} \mathrm{O}_{2}$ treatment. $\mathrm{MKN}-45$ cells were purchased from the Japanese Collection of Research Bioresources (Osaka, Japan) and maintained in RPMI-1640 (Sigma) supplemented with $10 \%$ fetal bovine serum and $1 \%$ penicillin and streptomycin in a humidified $5 \% \mathrm{CO}_{2}$ incubator. For the oxidative stress experiment, $5.5 \times 10^{5}$ cells were plated on a $10-\mathrm{cm}$ dish and cultured for 3 days in standard medium. Medium was aspirated and washed with phosphate-buffered saline (PBS) and cells were exposed to the indicated concentrations of $\mathrm{H}_{2} \mathrm{O}_{2}$ ranging from 0 to $2 \mathrm{mM}$ in the serum-free medium for $90 \mathrm{~min}$. Following exposure to oxidative stress, these cells were washed twice with ice-cold PBS and lysed in sample buffer [50 mM Tris- $\mathrm{HCl}$ (pH 6.8), $10 \%$ glycerol, $4 \%$ SDS and $0.01 \%$ bromophenol blue]. For reduction of disulfide links, $50 \mathrm{mM}$ DTT was added to the sample prior to electrophoresis. The samples were separated on $15 \%$ Tricine SDS-PAGE gels.

Plasmid transfection and pull down experiment. MKN-45 cells were cultured in a 60-mm dish and pME18S-FLAG-PP5 plasmid $(2 \mu \mathrm{g})$ was transfected using FuGENE HD transfection reagent according to the manufacturer's instructions (Roche Applied Science, Indianapolis, IN, USA). After $48 \mathrm{~h}$, cells were treated with or without $2 \mathrm{mM} \mathrm{H}_{2} \mathrm{O}_{2}$ and $0.5 \mu \mathrm{M}$ ionomycin for $90 \mathrm{~min}$. Cells were washed once with PBS and lysed in a buffer consisting of $50 \mathrm{mM}$ Tris- $\mathrm{HCl}$ (pH 7.5), $150 \mathrm{mM} \mathrm{NaCl}, 0.5 \%$ Triton X-100, and $0.5 \%$ Nonidet $\mathrm{P}-40$ with protease inhibitor mixture (Roche). The samples were sonicated and centrifuged at $16,600 \mathrm{x}$ g for $10 \mathrm{~min}$. Supernatants were incubated with $30 \mu 1$ of anti-FLAG antibody-agarose in the presence of $1 \mathrm{mM} \mathrm{CaCl}{ }_{2}$ for $2 \mathrm{~h}$ at room temperature. Following extensive washing, the beads were used either for the western blotting or in vitro phosphatase assay.

Surface plasmon resonance (SPR). Protein binding analysis was performed using an SPR Biacore 2000 system (GE Healthcare Institute, Waukesha, WI, USA). N-ethyl-N'-(3-diethylaminopropyl) carbodiimide, $\mathrm{N}$-hydroxysuccinimide and ethanolamine-HCl (GE Healthcare) were used for amine coupling of PP5 or S100A1 to the dextran surface of the CM5 chip. His-PP5 [2500 RU (pH 4.2)] or S100A1 [1400 RU (pH 4.5)] were immobilized in $10-\mathrm{mM}$ ammonium acetate. For all the procedures, HBS-P buffer [20 mM HEPES (pH 7.4), $150 \mathrm{mM} \mathrm{NaCl}$ and $0.005 \%$ Tween-20] with $1 \mathrm{mM} \mathrm{CaCl}{ }_{2}$ were used at a flow rate of $20 \mu \mathrm{l} / \mathrm{min}$. Various concentrations of recombinant S100 proteins were injected. The ligand-coupled sensor chips were regenerated between protein injections with a brief $(60 \mathrm{sec})$ wash with HBS-P buffer containing $2.5 \mathrm{mM}$ ethyleneglycolbis-(2-aminoethylether)-tetra acetic acid (EGTA) and 0.75\% n-octyl- $\beta$-D-glucopyranoside. Response curves were prepared for fitting by subtraction of the signal generated simultaneously on the control flow cell. Biacore sensorgrams were analyzed using BIAevaluation 4.1 software (GE Healthcare).

Native PAGE, Tricine SDS-PAGE and western blotting. Native PAGE analysis was performed based on a previous study (25). Tricine SDS-PAGE gel electrophoresis was performed under reducing and non-reducing conditions. The gels were either stained with Coomassie Brilliant Blue (CBB) or used for western blotting. For western blot analysis, proteins were blotted onto nitrocellulose membranes and blocked with 5\% skimmed milk in Tris-buffered saline with $0.05 \%$ Tween-20 (TTBS). The membranes were incubated with an anti-S100 antibody in the same buffer overnight. Membranes were washed with TTBS and incubated with an HRP-labeled second antibody for $2 \mathrm{~h}$. To detect the FLAG-tagged PP5, the anti-FLAG-HRP antibody was used. The samples were washed and signals were detected using Immobilon Western Chemiluminescent HRP Substrate (Millipore, Billerica, MA, USA).

In vitro phosphatase assay. Phosphatase activity of PP5 was measured using a Ser/Thr Phosphatase Assay kit (Upstate Biotechnology, Lake Placid, NY, USA) in accordance with the manufacturer's instructions. The phosphopeptide (KRpTIRR; $100 \mu \mathrm{M}$ ) was incubated with $250 \mathrm{ng}$ of His-PP5 in a buffer consisting of $20 \mathrm{mM}$ Tris- $\mathrm{HCl}$ (pH 7.5), $20 \mathrm{mM} \mathrm{MgCl} 2,0.01 \%$ Tween-20 with $1 \mathrm{mM} \mathrm{CaCl}{ }_{2}$ or EGTA in a volume of $50 \mu 1$. Various amounts $(0-10 \mu \mathrm{g})$ of S100 proteins were added and incubated for $10 \mathrm{~min}$ at $37^{\circ} \mathrm{C}$. Following the addition of $100 \mu \mathrm{l}$ of malachite green solution $(0.034 \%$ malachite green, $10 \mathrm{mM}$ ammonium molybdate, $1 \mathrm{M} \mathrm{HCl}, 3.4 \%$ ethanol and $0.01 \%$ Tween-20), the absorbance of samples at $630 \mathrm{~nm}$ was 
measured using a microplate reader. The amount of released phosphate was calculated using a phosphate standard curve prepared from a known amount of phosphate. To measure the PP5 activity bound to the FLAG-agarose beads, $10 \mu \mathrm{l}$ of beads (in triplicate) were used and incubated in the presence of $1 \mathrm{mM} \mathrm{CaCl}_{2}$ for $1 \mathrm{~h}$ at $37^{\circ} \mathrm{C}$. The samples were centrifuged and supernatants were used for the assay.

Statistical analysis. Statistical analysis was performed using the Student's t-test and $\mathrm{P}<0.05$ was considered to indicate a statistically significant difference. Data are expressed as the means \pm standard deviation.

\section{Results}

Oxidation of S100A4 promotes oligomerization by intermolecular disulfide bridge formation and inhibits PP5 activation by $S 100 A 1$. The preliminary experiments indicated that the effects of S100A4 on S100A1-stimulated PP5 activity varied from sample to sample (i.e., duration of storage of S100A4 preparation). It was suspected that the sample-to-sample variation depended on the oxidation level of S100A4 during storage. Recombinant S100A4 protein was purified from E. coli and the protein was immediately used as a freshly prepared sample. Air-oxidized S100A4 was prepared by maintaining the diluted protein $(0.5 \mathrm{mg} / \mathrm{ml})$ in a freezer at $-30^{\circ} \mathrm{C}$ for three months in order to promote oxidation. Air-oxidized and freshly prepared samples were separated with Tricine SDS-PAGE gel under reducing (with DTT) or non-reducing (without DTT) conditions, followed by staining with CBB (Fig. 1A). With or without DTT treatment, the majority of proteins were observed as monomers and a small portion was observed as dimers in the freshly prepared S100A4 lanes. Without DTT, air-oxidation decreased the monomeric form and increased the dimeric form. In addition, smear bands were observed above the dimer band that could represent higher molecular weight (HMW) forms. Treatment with DTT decreased the dimeric and HMW forms, while the amount of monomer increased. These results indicate that oxidation of S100A4 promoted oligomerization by intermolecular disulfide bridge formation. Subsequently, the PP5 activation assay was performed (Fig. 1B). The basic activity of PP5 is kept extremely low (26). In the presence of $\mathrm{Ca}^{2+}, \mathrm{S} 100 \mathrm{~A} 1$ significantly activated PP5 $(593.7 \pm 32.0 \mathrm{nmol} / \mathrm{min} / \mathrm{ng}$ protein with $10 \mu \mathrm{g}$ of S100A1), but no clear activation was observed in the presence of EGTA. When a fixed amount of S100A1 $(2.5 \mu \mathrm{g})$ and increasing amounts of freshly prepared S100A4 were used, S100A4 showed no clear effects on S100A1 activated PP5 activity in the presence of $\mathrm{Ca}^{2+}$ (Fig. 1C). Freshly prepared S100A4 itself could not activate PP5, even in the presence of $\mathrm{Ca}^{2+}$. However, addition of air-oxidized S100A4 to the fixed amount of S100A1 dose-dependently inhibited activation of PP5 by S100A1 in the presence of $\mathrm{Ca}^{2+}$ (Fig. 1D). No activation was observed with the air-oxidized S100A4.

Air-oxidized S100A4 inhibits the interaction between PP5 and S100A1. In order to understand the mechanism through which oxidized S100A4 inhibits PP5 activation by S100A1, SPR analysis was performed to measure the interaction between S100A4 or S100A1 and PP5 (Fig. 2). To determine whether

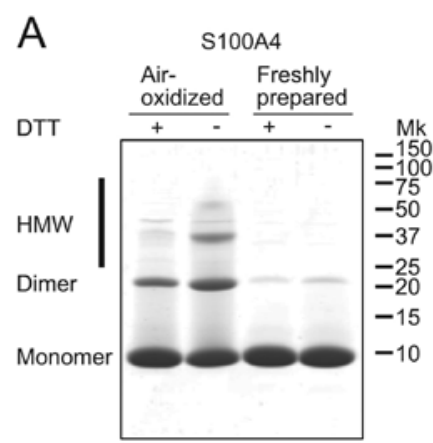

B

C
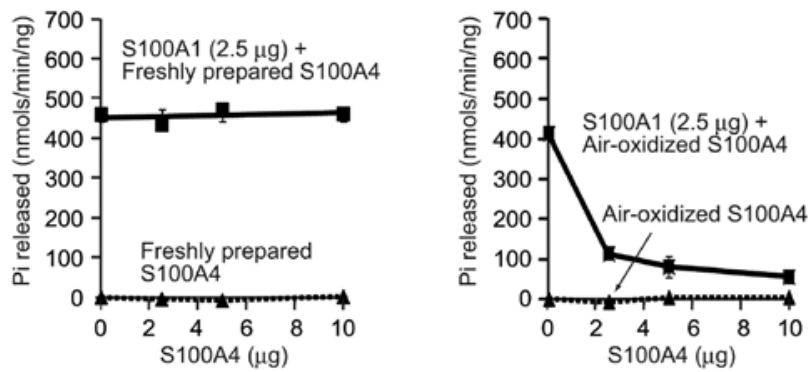

Figure 1. Oxidation of S100A4 and its effect on PP5 activation by S100A1. (A) Air-oxidized and freshly prepared S100A4 were separated on a $15 \%$ Tricine SDS-PAGE gel under reducing [dithiothreitol (DTT) (+)] or non-reducing [(DTT(-)] conditions, followed by staining with Coomassie Brilliant Blue (CBB). HMW, high molecular weight complex. Molecular weight markers are shown on the right $(\mathrm{kDa})$. (B) PP5 activation assay. His-PP5 (250 ng) was mixed with $100 \mu \mathrm{M}$ phosphopeptide and various amounts of S100A1 in the presence of $1 \mathrm{mM} \mathrm{CaCl}_{2}$ or EGTA, followed by incubation for $10 \mathrm{~min}$ at $37^{\circ} \mathrm{C}$. Malachite green solution with $0.01 \%$ Tween-20 was added and absorbance at $630 \mathrm{~nm}$ was measured. The amount of released phosphate was determined using a standard curve calculated from a known amount of phosphate. Each point represents the mean \pm standard deviation (SD) of triplicate determinations. Fixed amounts $(2.5 \mu \mathrm{g})$ of S100A1 and various amounts of (C) freshly prepared S100A4 or (D) air-oxidized S100A4 were used for the PP5 activation assay in the presence of $1 \mathrm{mM} \mathrm{CaCl}_{2}$. Each point represents the mean \pm SD of triplicate determinations.

a positive interaction existed between S100A1 and freshly prepared S100A4, individual binding experiments were first performed for saturating amounts of each protein $(10 \mu \mathrm{M})$ using a PP5-coupled chip (Fig. 2A). There were larger amounts of freshly prepared S100A4 bound to PP5, as compared to S100A1 ( 330 RU of S100A4 and 159 RU of S100A1 at $150 \mathrm{sec})$. In the second experiment, S100A1 was first injected over the PP5-coupled chip, and following association with S100A1, freshly prepared S100A4 was added using the COINJECT function of the Biacore instrument. Under these reaction conditions (Fig. 2B), the sum of the interactions appeared to be additive. When the order of addition was reversed (S100A1 and freshly prepared S100A4 were injected first, followed by S100A1), the binding events were found to be additive for PP5 (Fig. 2C). These results indicated that the proteins bind independently from one another, and that these two proteins may not physically interact. By contrast, air-oxidized S100A4 significantly lost its ability to bind PP5 (Fig. 2D) and notably, increased amounts of oxidized S100A4 inhibited the binding of S100A1 to PP5 in a dose-dependent manner (Fig. 2E). This result agreed with the inhibitory effect of the oxidized 

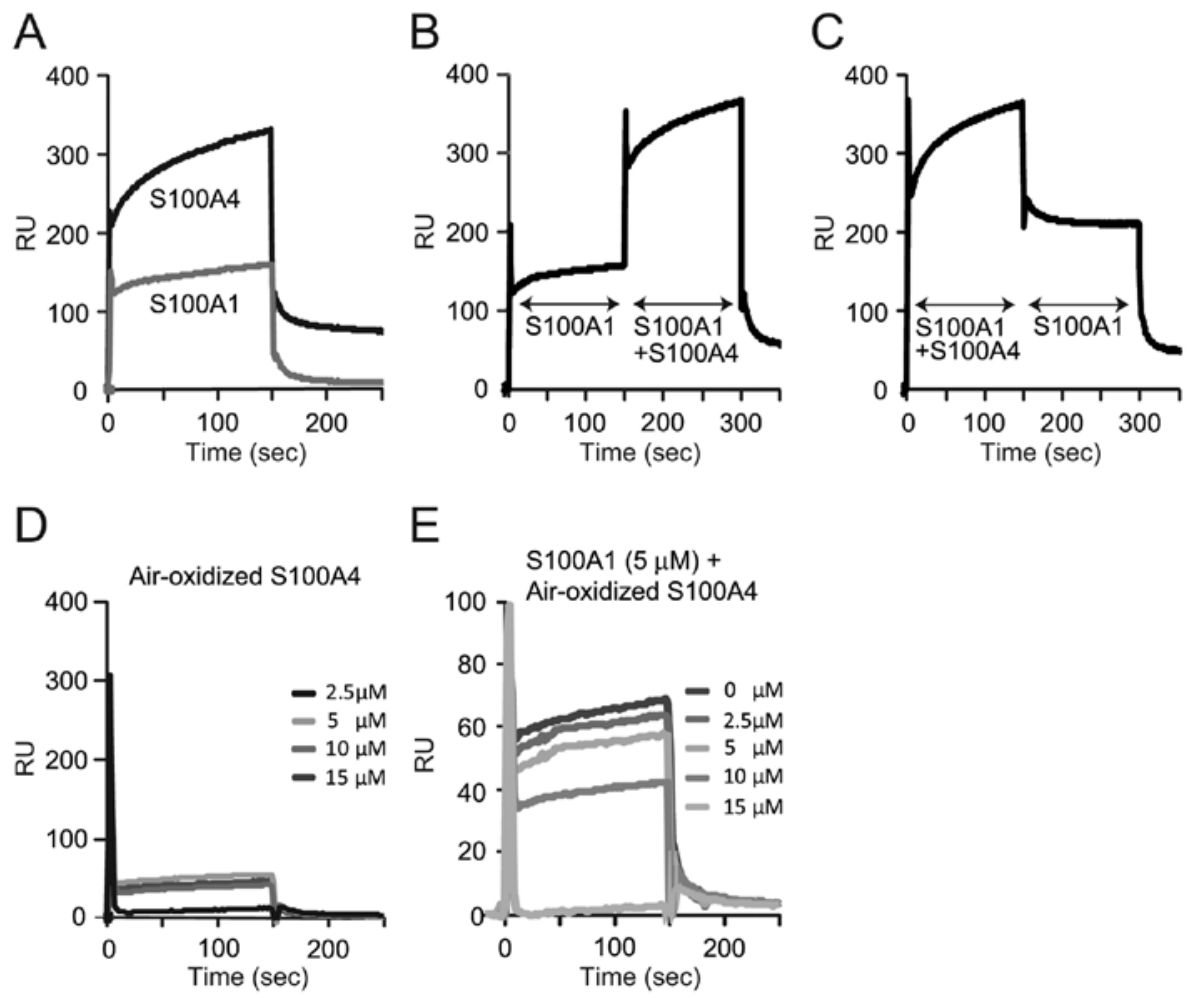

Figure 2.Interaction between PP5 and S100 proteins.Protein binding analysis was performed using a SPR Biacore 2000 system. $N$-ethyl- $N$ '-(3-diethylaminopropyl) carbodiimide, $N$-hydroxysuccinimide, and ethanolamine- $\mathrm{HCl}$ (Biacore) were used for amine coupling of PP5 or S100A1 to the dextran surface of the CM5 chip. His-PP5 [2500 RU (pH 4.2)] or S100A1 [1400 RU (pH 4.5)] was immobilized in 10-mM ammonium acetate. Various concentrations of recombinant S100 proteins were injected. Response curves were prepared for fitting by subtraction of the signal generated simultaneously on the control flow cell. Biacore sensorgrams were analyzed using BIAevaluation 4.1 software. (A) Freshly prepared S100A1 or S100A4. (B) Freshly prepared S100A1 firstly, followed by injection of S100A1 and S100A4. (C) S100A1 and S100A4 were injected, followed by S100A1 alone. (D) Various concentrations of air-oxidized S100A4 were injected. (E) Fixed amount $(5 \mu \mathrm{M})$ of S100A1 and various concentrations of air-oxidized S100A4 were injected.

S100A4 on the PP5 activation by S100A1 (Fig. 1D). These results indicate that oxidized S100A4 prevented S100A1 from PP5 activation by forming complexes with S100A1, but not by competitively disturbing the interaction between PP5 and S100A1.

Cu-oxidized S100A4 fails to interact with PP5 and inhibits interaction between PP5 and S100A1. The effects of oxidized S100A4 were further examined on PP5 activation by copper oxidation. Freshly prepared and $\mathrm{Cu}$-oxidized S100A4 was separated with Tricine SDS-PAGE gel under reducing (with DTT) or non-reducing (without DTT) conditions and stained with CBB (Fig. 3A). Freshly prepared S100A4 formed a monomer and a small portion was dimeric, as shown in Fig. 1A. Without DTT, Cu oxidation significantly decreased the monomeric form and increased the dimeric and HMW forms. Treatment with DTT decreased the dimeric and HMW forms, while the amount of monomer increased. The PP5 activation assay was also performed. $\mathrm{Cu}$-oxidized S100A4 was unable to activate PP5, and addition of the $\mathrm{Cu}$-oxidized S100A4 to a fixed amount of S100A1 (2.5 $\mu \mathrm{g} /$ tube $)$ dose-dependently inhibited activation of PP5 by S100A1 in the presence of $\mathrm{Ca}^{2+}$ (Fig. 3B). A similar inhibitory effect was observed with air-oxidized S100A4 in Fig. 1D. Using the PP5 immobilized chip, Cu-oxidized S100A4 failed to bind PP5 (Fig. 3D), whereas the dose-dependent binding of freshly prepared S100A4 was observed by SPR (Fig. 3C). When
S100A1 was immobilized on the chip, freshly prepared S100A4 bound only slightly to the S100A1-coupled chip (Fig. 3E). Notably, the oxidation of S100A4 clearly increased its affinity for S100A1 (Fig. 3F). The binding of Cu-oxidized S100A4 to S100A1 increased 3 -fold (90 RU at $150 \mathrm{sec}$ ) compared to that of freshly prepared S100A4 (33 RU at $150 \mathrm{sec})$.

Native PAGE analysis of S100 proteins and PP5 interaction. In order to confirm SPR experiments, a non-denaturing gel shift assay previously used for calmodulin $(\mathrm{CaM})$ binding proteins was employed $(27,28)$. In the presence of $\mathrm{Ca}^{2+}, \mathrm{S} 100 \mathrm{~A} 1$ was mainly observed as a dimer and a faint multimer band (Fig. 4A and B). As has been previously reported (29), freshly prepared S100A4 was detected near the origin of the gel as a broad band. Cu-oxidized S100A4 formed a broad band, but the electrophoretic mobility was different from that of freshly prepared S100A4. PP5 was unable to migrate into gels, and was observed as a sharp band on the top of the separating gel. Possible binding between the S100 proteins and PP5 was subsequently tested, in which mixtures of the two were run on non-denaturing polyacrylamide gels. Mixtures of S100A1 and PP5 formed new multiple bands (indicated by closed arrow heads), thus indicating the interaction between these proteins. By contrast, addition of freshly prepared S100A4 and $\mathrm{Cu}$-oxidized S100A4 did not alter PP5 mobility in the presence of $\mathrm{Ca}^{2+}$. 

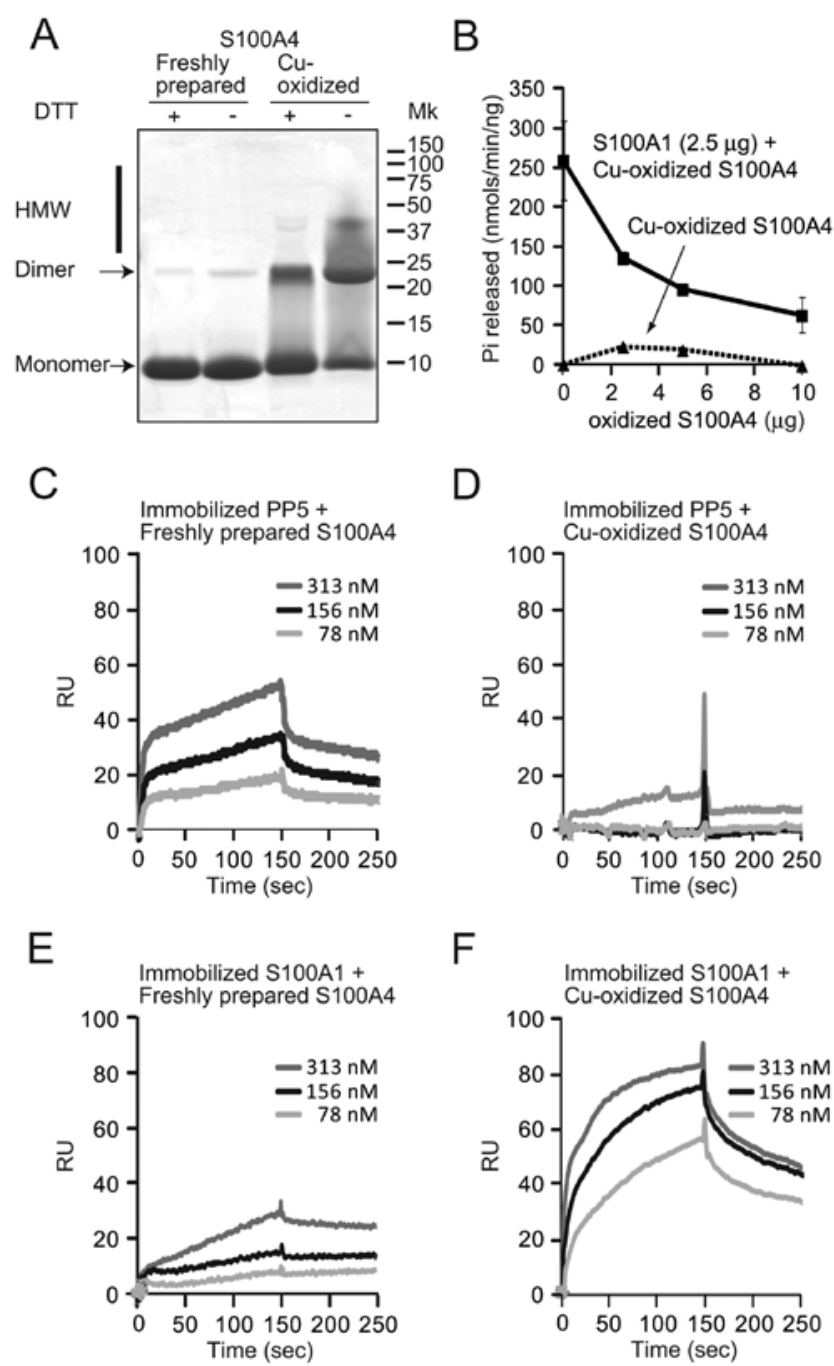

Figure 3. Effects of Cu-oxidized S100A4 on the interaction with PP5 or S100A1 and PP5 activation. (A) Freshly prepared and Cu-oxidized S100A4 were separated on $15 \%$ Tricine SDS-PAGE gels under reducing [dithiothreitol (DTT) (+)] or non-reducing [(DTT(-)] conditions, and were stained with Coomassie Brilliant Blue (CBB). HMW, high molecular weight complex. Molecular weight markers are shown on the right $(\mathrm{kDa})$. (B) Various amounts of $\mathrm{Cu}$-oxidized S100A4 with or without fixed amount of S100A1 $(2.5 \mu \mathrm{g})$ were used for the PP5 activation assay in the presence of $1 \mathrm{mM} \mathrm{CaCl}_{2}$. Each point represents the mean \pm standard deviation $(\mathrm{SD})$ of triplicate determinations. (C) His-PP5 was immobilized on the sensor chip and various amounts of freshly prepared S100A4 were applied. Binding was measured by surface plasmon resonance (SPR). (D) The binding of Cu-oxidized S100A4 onto the PP5-immobilized chip was measured. (E) S100A1 was immobilized on the sensor chip and various amounts of freshly prepared S100A4 were applied. Binding was measured by SPR analysis. (F) Binding of $\mathrm{Cu}$-oxidized S100A4 to the S100A1 immobilized on the chip was measured.

Subsequently, the interaction between S100A4 and S100A1 was examined (Fig. 4B). Cu-oxidized S100A4 caused a notable shift in the electrophoretic mobility of S100A1 (indicated open arrowheads), whereas freshly prepared S100A4 did not. Taken together, these results indicate that $\mathrm{Cu}$-oxidized S100A4, but not freshly prepared S100A4, directly interact with S100A1.

Oxidative stress promotes oligomerization of S100A4 by intermolecular disulfide bridge formation in $M K N-45$ cells. A previous study indicated that treatment with $\mathrm{H}_{2} \mathrm{O}_{2}$ strongly promoted intermolecular disulfide cross-linking of naturally expressed
A

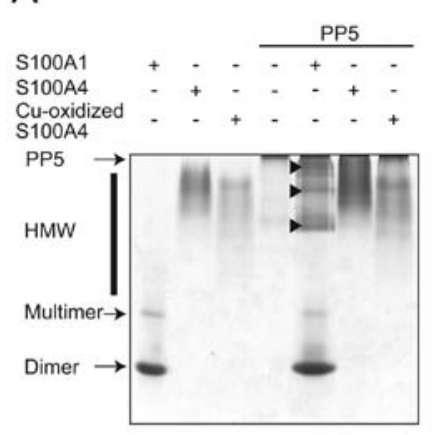

B

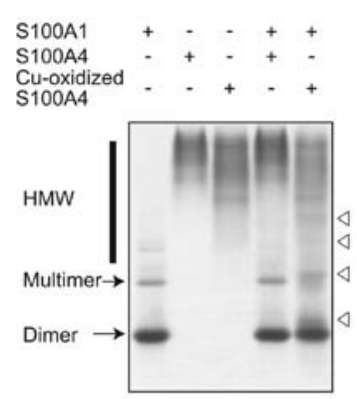

C
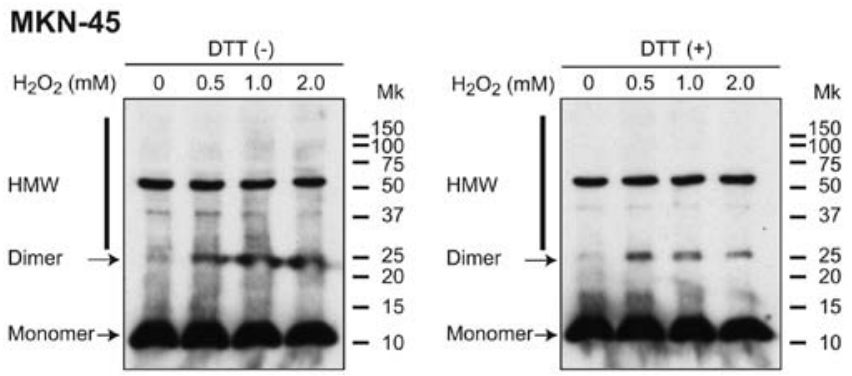

Figure 4. Native PAGE analysis of S100-PP5 interaction and the effect of $\mathrm{H}_{2} \mathrm{O}_{2}$ on S100A4 in MKN-45 cells. (A) Approximately $10 \mu \mathrm{g}$ each of S100A1, S100A4 or Cu-oxidized S100A4 was separated on the gel (left three lanes). Same samples were incubated with PP5 and separated on the same gel (right three lanes). Gels were stained with Coomassie Brilliant Blue (CBB). Multiple bands formed by mixture of S100A1 and PP5 are indicated by closed arrow heads. (B) Approximately $10 \mu \mathrm{g}$ each of freshly prepared S100A1, S100A4, $\mathrm{Cu}$-oxidized S100A4, S100A1 + S100A4 or S100A1 + Cu-oxidized S100A4 were applied to the gels. Gels were stained with CBB. Electrophoretic mobility shifts of S100A1 by $\mathrm{Cu}$-oxidized S100A4 are indicated (open arrowhead). Multiple bands formed by mixture of S100A1 and PP5 are indicated by closed arrow heads. (C) Approximately $5.5 \times 10^{5}$ cells were plated on a $10-\mathrm{cm}$ dish and cultured for 3 days in standard medium. Medium was aspirated and washed with PBS and cells were exposed to the indicated concentrations of $\mathrm{H}_{2} \mathrm{O}_{2}$ ranging from 0 to $2 \mathrm{mM}$ in the serum-free medium for $90 \mathrm{~min}$. Proteins were extracted and separated on 15\% Tricine SDS-PAGE under reducing [dithiothreitol (DTT) (+)] or non-reducing [(DTT(-)]conditions. Western blot analysis was performed using an anti-S100A4 antibody. HMW, high molecular weight complexes. The band migrating with an apparent MW of $60 \mathrm{kDa}$ is a non-specific horseradish peroxidase (HRP)-labeled secondary antibody-reactive band. Molecular weight markers are shown on the right $(\mathrm{kDa})$.

S100A2 in human keratinocytes or HaCaT cells (30). According to the reported procedure (21), whether oxidative stress promotes the intermolecular disulfide cross-linking of S100A4 in the intact cells was examined. MKN-45 gastric adenocarcinoma cells were used as this cell line is often used for the oxidative stress study and the expression of S100A4 was confirmed (31). Following treatment with various concentrations of $\mathrm{H}_{2} \mathrm{O}_{2}$, cells were lysed in SDS-PAGE sample buffer without any reducing agents. The samples were treated with or without DTT and separated on Tricine SDS-PAGE gels. S100A4 was detected by western blotting with an anti-S100A4 antibody (Fig. 4C). $\mathrm{H}_{2} \mathrm{O}_{2}$ treatment increased the formation of dimeric S100A4 and HMW complexes in a dose-dependent manner (Fig. 4C, left panel). Addition of DTT clearly reduced the formation of these forms (Fig. 4C, right panel), indicating that the intermolecular disulfide cross-linking of S100A4 occurs in intact cells. 


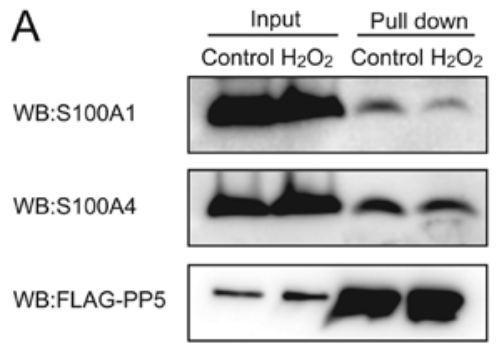

B

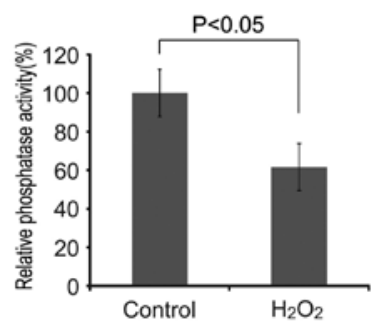

Figure 5. Effect of oxidative stress on S100A1-PP5 interaction and PP5 activation in MKN-45 cells. (A) MKN-45 cells were transfected with pME18S-FLAG-PP5 plasmid. The cells were treated with or without $2 \mathrm{mM}$ $\mathrm{H}_{2} \mathrm{O}_{2}$ and $0.5 \mu \mathrm{M}$ ionomycin for $90 \mathrm{~min}$. The pull down experiment was performed using $400 \mu \mathrm{g}$ of total protein and anti-FLAG agarose. Following intensive washes, the samples were separated with Tricine SDS-PAGE gels and western blotting was carried out using indicated antibodies. Approximately $20 \mu \mathrm{g}$ of protein was used as an input. (B) Phosphatase activity of FLAG-agarose bound PP5 was measured and relative phosphatase activity was calculated. Data are expressed as the mean \pm standard deviation $(\mathrm{SD})(\mathrm{n}=3)$.

Oxidized S100A4 inhibits the activation of PP5 by S100AI in MKN-45 cells. Subsequently, the effect of $\mathrm{H}_{2} \mathrm{O}_{2}$ on S100A1-PP5 interaction and PP5 activation was examined. FLAG-tagged PP5 was expressed in the MKN-45 cells and cells were treated with or without $2 \mathrm{mM} \mathrm{H}_{2} \mathrm{O}_{2}$ and $0.5 \mu \mathrm{M}$ ionomycin for $90 \mathrm{~min}$. The result of the pull down experiment showed that $\mathrm{H}_{2} \mathrm{O}_{2}$ treatment decreased the S100A1 binding to PP5 (Fig. 5A). In addition, the PP5 activity was inhibited to $61.6 \pm 12.1 \%$ of the control $(\mathrm{P}<0.05$; Fig. $5 \mathrm{~B})$. Together with the results of Figs. 3 and $4 \mathrm{C}$, this data indicated that oxidized S100A4 prevented the binding of S100A1 to PP5, resulting in the inhibition of PP5 activation.

\section{Discussion}

S100A4 exerts its functions mainly through interaction with other proteins. Similar to other S100 proteins, a number of target proteins for S100A4 have been identified, including actin, non-muscle myosin IIA and IIB, tropomyosin, p53, liprin $\beta 1$, methionine aminopeptidase $2, \mathrm{CCN} 3, \mathrm{p} 37$, and septin 2, 6 and 7 (3-6,32-34). However, the functional correlations between S100A4-target interactions have not been widely described. In the extracellular space, S100A4 has been reported to interact with the receptor for advanced glycation end products (RAGE) and heparan sulfate proteoglycans $(35,36)$. In general, the biological activity of extracellular S100A4 is associated with multimeric forms of the protein. In the intracellular space, S100A4 binds to the targets in a $\mathrm{Ca}^{2+}$-dependent manner and regulates cellular functions. An increase of intracellular $\mathrm{Ca}^{2+}$ concentration promotes the non-covalent dimerization of S100A4 (8). As well as homodimer formation, two-hybrid screening detected the S100A4-S100A1 heterodimer $(9,10)$, and the interaction was detected in HeLa cells by fluorescence resonance energy transfer (10). In addition, oxidative modifications of S100 proteins induced their conformational changes and altered the functions. Several S100 proteins, such as S100A2, S100A3 or S100A4, have highly reactive cysteine residues at the surface that oxidize readily and form disulfide bonds (37). The present study demonstrated that air- and $\mathrm{Cu}$-oxidation of S100A4 promoted the formation of covalently (disulfide)-linked dimer or HMW complexes (Fig. 1A). Of note, oxidation of S100A4 markedly decreased the affinity to PP5 (Figs. 2D and 3D), but increased its affinity to S100A1 (Fig. 3F). This could result from oligomerization of S100A4 by intermolecular disulfide bridge formation, not from oligomerization under normal reducing conditions. Under normal circumstances, the TPR domain covers the catalytic domain of the enzyme and maintains a low activity (26). Although S100A1 and S100A4 are able to bind to the TPR domain of PP5 in a $\mathrm{Ca}^{2+}$-dependent manner, only S100A1 uncovers the catalytic domain, resulting in enzyme activation. SPR analysis in Fig. 2B and C supports the idea that S100A1 and S100A4 bind to a different region in PP5. When ROS are generated and the redox signaling pathway is activated, oxidized S100A4 binds to S100A1 and prevents PP5 activation. Although the detailed structure of the oxidized S100A4 is unknown, dimerization or oligomerization of S100A4 by intermolecular disulfide bridge formation concealed the interaction site with the TPR domain of PP5 and exposed the S100A1-interacting domain. Oxidation of S100A4 and inhibition of PP5 activation by S100A1 was observed in MKN-45 gastric adenocarcinoma cells following $\mathrm{H}_{2} \mathrm{O}_{2}$ treatment (Figs. $4 \mathrm{C}$ and $5 \mathrm{~B}$ ), thus, indicating that $\mathrm{S} 100 \mathrm{~A} 4$ could be oxidized under the strong oxidative stress conditions and may modulate signaling pathways inside the cells. A few studies have described the function of oxidized S100A4 in the extracellular space; the oligomeric forms of S100A4 strongly induced differentiation of cultured hippocampal neurons (38), and copper-mediated cross-linking of S100A4 increased NF- $\mathrm{NB}$ activation and TNF- $\alpha$ secretion in human A375 and in RAGE-transfected melanoma cells (39). By contrast, the role of oxidized S100A4 in the intracellular space is limited. The present results indicate that S100A4 is able to modulate the activity of PP5 on oxidative stress. PP5 is a negative regulator of ASK1, and PP5 activation by S100A1 and de-activation by oxidized S100A4 modulated apoptosis by regulating ASK1 activity under oxidative stress conditions. As gastric epithelium is exposed to ROS generated by food, cigarette smoke and inflammation by Helicobacter pylori infection that activates ASK1 under oxidative conditions, modulation of apoptosis could be important for the maintenance of mucosal homeostasis. Further study is required to help to understand the role of S100 proteins on gastric mucosa maintenance or tumor progression under oxidative stress conditions.

\section{Acknowledgements}

The present study was supported by the Kagawa University Characteristic Prior Research Fund 2011 (Kagawa, Japan). The authors would like to thank Keiko Tsurumi (a laboratory technician; Kagawa University) for technical assistance. 


\section{References}

1. Schäfer BW and Heizmann CW: The S100 family of EF-hand calcium-binding proteins: functions and pathology. Trends Biochem Sci 21: 134-140, 1996.

2. Santamaria-Kisiel L, Rintala-Dempsey AC and Shaw GS Calcium-dependent and -independent interactions of the S100 protein family. Biochem J 396: 201-214, 2006.

3. Kriajevska M, Fischer-Larsen M, Moertz E, et al: Liprin beta 1 , a member of the family of LAR transmembrane tyrosine phosphatase-interacting proteins, is a new target for the metastasis-associated protein S100A4 (Mts1). J Biol Chem 277: 5229-5235, 2002.

4. Endo H, Takenaga K, Kanno T, Satoh H and Mori S: Methionine aminopeptidase 2 is a new target for the metastasis-associated protein, S100A4. J Biol Chem 277: 26396-26402, 2002.

5. Garrett SC, Varney KM, Weber DJ and Bresnick AR: S100A4, a mediator of metastasis. J Biol Chem 281: 677-680, 2006.

6. Kiss B, Duelli A, Radnai L, Kékesi KA, Katona G and Nyitray L: Crystal structure of the S100A4-nonmuscle myosin IIA tail fragment complex reveals an asymmetric target binding mechanism. Proc Natl Acad Sci USA 109: 6048-6053, 2012.

7. Tarabykina S, Kriajevska M, Scott DJ, et al: Heterocomplex formation between metastasis-related protein S100A4 (Mts1) and S100A1 as revealed by the yeast two-hybrid system. FEBS Lett 475: 187-191, 2000

8. Tarabykina S, Scott DJ, Herzyk P, et al: The dimerization interface of the metastasis-associated protein S100A4 (Mts1): in vivo and in vitro studies. J Biol Chem 276: 24212-24222, 2001

9. Wang G, Rudland PS, White MR and Barraclough R: Interaction in vivo and in vitro of the metastasis-inducing S100 protein S100A4 (p9Ka) with S100A1. J Biol Chem 275: 11141-11146, 2000.

10. Wang G, Zhang S, Fernig DG, Martin-Fernandez M, Rudland PS and Barraclough R: Mutually antagonistic actions of S100A4 and S100A1 on normal and metastatic phenotypes. Oncogene 24 1445-1454, 2005

11. Deloulme JC, Gentil BJ and Baudier J: Monitoring of S100 homodimerization and heterodimeric interactions by the yeast two-hybrid system. Microsc Res Tech 60: 560-568, 2003.

12. Pröpper C, Huang X, Roth J, Sorg C and Nacken W: Analysis of the MRP8-MRP14 protein-protein interaction by the two-hybrid system suggests a prominent role of the C-terminal domain of S100 proteins in dimer formation. J Biol Chem 274: 183-188, 1999.

13. Wang G, Zhang S, Fernig DG, et al: Heterodimeric interaction and interfaces of S100A1 and S100P. Biochem J 382: 375-383, 2004.

14. Yamaguchi F, Umeda Y, Shimamoto S, et al: S100 protein modulate protein phosphatase 5 function: a link between $\mathrm{CA}^{2+}$ signal transduction and protein dephosphorylation. J Biol Chem 287: 13787-13798, 2012.

15. Hinds TD Jr and Sánchez ER: Protein phosphatase 5. Int J Biochem Cell Biol 40: 2358-2362, 2008.

16. Becker W, Kentrup H, Klumpp S, Schultz JE and Joost HG Molecular cloning of a protein serine/threonine phosphatase containing a putative regulatory tetratricopeptide repeat domain. J Biol Chem 269: 22586-22592, 1994.

17. Chen MX, McPartlin AE, Brown L, Chen YH, Barker HM and Cohen PT: A novel human protein serine/threonine phosphatase, which possesses four tetratricopeptide repeat motifs and localizes to the nucleus. EMBO J 13: 4278-4290, 1994.

18. D'Andrea LD and Regan L: TPR proteins: the versatile helix. Trends Biochem Sci 28: 655-662, 2003.

19. Zeytuni N and Zarivach R: Structural and functional discussion of the tetra-trico-peptide repeat, a protein interaction module. Structure 20: 397-405, 2012.

20. Morita K, Saitoh M, Tobiume K, et al: Negative feedback regulation of ASK1 by protein phosphatase 5 (PP5) in response to oxidative stress. EMBO J 20: 6028-6036, 2001.
21. Gencer S and Irmak Yazicioğlu MB: Differential response of gastric carcinoma MKN-45 and 23132/87 cells to $\mathrm{H}_{2} \mathrm{O}_{2}$ exposure. Turk J Gastroenterol 22: 145-151, 2011.

22. Okada M, Hatakeyama T, Itoh H, Tokuta N, Tokumitsu H and Kobayashi R: S100A1 is a novel molecular chaperone and a member of the Hsp70/Hsp90 multichaperone complex. J Biol Chem 279: 4221-4233, 2004.

23. Yamashita K, Oyama Y, Shishibori T, Matsushita O, Okabe A and Kobayashi R: Purification of bovine S100A12 from recombinant Escherichia coli. Protein Expr Purif 16: 47-52, 1999.

24. Matsui Lee IS, Suzuki M, Hayashi N, et al: Copper-dependent formation of disulfide-linked dimer of S100B protein. Arch Biochem Biophys 374: 137-141, 2000.

25. Okada M, Tokumitsu H, Kubota Y and Kobayashi R: Interaction of S100 proteins with the antiallergic drugs, olopatadine, amlexanox, and cromolyn: identification of putative drug binding sites on S100A1 protein. Biochem Biophys Res Commun 292: 1023-1030, 2002

26. Chinkers M: Protein phosphatase 5 in signal transduction. Trends Endocrinol Metab 12: 28-32, 2001.

27. Zühlke RD, Pitt GS, Deisseroth K, Tsien RW and Reuter H: Calmodulin supports both inactivation and facilitation of L-type calcium channels. Nature 399: 159-162, 1999.

28. Takata M, Shimamoto S, Yamaguchi F, Tokuda M, Tokumitsu H and Kobayashi R: Regulation of nuclear localization signal-importin alpha interaction by $\mathrm{Ca} 2+/ \mathrm{S} 100 \mathrm{~A} 6$. FEBS Lett 584: 4517-4523, 2010.

29. Gibbs FE, Wilkinson MC, Rudland PS and Barraclough R: Interactions in vitro of $\mathrm{p} 9 \mathrm{Ka}$, the rat $\mathrm{S}-100$-related, metastasis-inducing, calcium-binding protein. J Biol Chem 269: 18992-18999, 1994.

30. Zhang T, Woods TL and Elder JT: Differential responses of S100A 2 to oxidative stress and increased intracellular calcium in normal, immortalized, and malignant human keratinocytes. J Invest Dermatol 119: 1196-1201, 2002.

31. Yonemura Y, Endou Y, Kimura K, et al: Inverse expression of S100A4 and E-cadherin is associated with metastatic potential in gastric cancer. Clin Cancer Res 6: 4234-4242, 2000.

32. Watanabe $\mathrm{Y}$, Usada $\mathrm{N}$, Minami $\mathrm{H}$, et al: Calvasculin, as a factor affecting the microfilament assemblies in rat fibroblasts transfected by src gene. FEBS Lett 324: 51-55, 1993.

33. Takenaga K, Nakamura Y, Sakiyama S, Hasegawa Y, Sato K and Endo H: Binding of pEL98 protein, an S100-related calcium-binding protein, to nonmuscle tropomyosin. J Cell Biol 124: 757-768, 1994.

34. Li CL, Martinez V, He B, Lombet A and Perbal B: A role for CCN3 (NOV) in calcium signalling. Mol Pathol 55: 250-261, 2002.

35. Yammani RR, Carlson CS, Bresnick AR and Loeser RF: Increase in production of matrix metalloproteinase 13 by human articular chondrocytes due to stimulation with S100A4: Role of the receptor for advanced glycation end products. Arthritis Rheum 54: 2901-2911, 2006.

36. Kiryushko D, Novitskaya V, Soroka V, et al: Molecular mechanisms of $\mathrm{Ca}^{2+}$ signaling in neurons induced by the S100A4 protein. Mol Cell Biol 26: 3625-3638, 2006.

37. Fritz G: X-ray structural analysis of S100 proteins. Methods Mol Biol 963: 87-97, 2013

38. Novitskaya V, Grigorian M, Kriajevska M, et al: Oligomeric forms of the metastasis-related Mts1 (S100A4) protein stimulate neuronal differentiation in cultures of rat hippocampal neurons. J Biol Chem 275: 41278-41286, 2000.

39. Haase-Kohn C, Wolf S, Lenk J and Pietzsch J: Copper-mediated cross-linking of S100A4, but not of S100A2, results in proinflammatory effects in melanoma cells. Biochem Biophys Res Commun 413: 494-498, 2011. 